\title{
Multi-scale Simulations of Gas Flows with Unified Flow Solver
}

\author{
V.V. Aristov ${ }^{1}$, A.A. Frolova ${ }^{1}$, S.A. Zabelok ${ }^{1}$, \\ V.I. Kolobov ${ }^{2}$, and R.R. Arslanbekov ${ }^{2}$ \\ ${ }^{1}$ Dorodnicyn Computing Center of the Russian Academy of Sciences \\ Vavilova str., 40, 119991, Moscow, Russia \\ \{aristov, afrol, serge\} accas.ru \\ ${ }^{2}$ CFD Research Corporation, 215 Wynn Drive, \\ Huntsville, AL , 35803, USA \\ \{vik, rra\}@efdrc.com
}

\begin{abstract}
The Boltzmann kinetic equation links micro- and macroscale descriptions of gases. This paper describes multi-scale simulations of gas flows using a Unified Flow Solver (UFS) based on the Boltzmann equation. A direct Boltzmann solver is used for microscopic simulations of the rarefied parts of the flows, whereas kinetic CFD solvers are used for the continuum parts of the flows. The UFS employs an adaptive mesh and algorithm refinement procedure for automatic decomposition of computational domain into kinetic and continuum parts. The paper presents examples of flow problems for different Knudsen and Mach numbers and describes future directions for the development of UFS technology.
\end{abstract}

Keywords: Boltzmann equation, Rarefied Gas Dynamics, direct Boltzmann solver, kinetic CFD scheme, multiscale flows, adaptive mesh.

\section{Introduction}

The Boltzmann kinetic equation is a fundamental physical tool, which links two levels of description of gases. The first one is the microscopic level at atomistic scale and the second one is the macroscopic level at continuum scale. The kinetic equation takes into account interactions among gas particles under the molecular chaos assumption to describe phenomena on the scale of the mean free path. In principle, the Boltzmann equation can also describe macroscopic phenomena, but its usage at the macroscopic scale becomes prohibitory expensive and not necessary. The continuum equations (Euler or Navier-Stokes) derived from the Boltzmann equations can be used at this scale. The present paper describes mathematical methods, which allow one to fully realize this property of the Boltzmann equation. The Unified Flow Solver (UFS) is a variant of hybrid methods for simulation of complex gas (or maybe liquid) flows in the presence of multiscale phenomena including nonequilibrium and nonlinear processes. The UFS methodology combines direct numerical solution of the Boltzmann equation with the kinetic schemes (asymptotic case of the direct methods of solving the Boltzmann equation), which approximate the Euler or Navier-Stokes (NS) equations. 
The development of hybrid solvers combining kinetic and continuum models has been an important area of research over the last decade (see Ref. [1] for review). Most researchers applied traditional DSMC methods for rarefied domains and identified statistical noise inherent to the particle methods as an obstacle for coupling kinetic and continuum solvers [2]. The UFS combines direct Boltzmann solver [3,4] with kinetic CFD schemes to facilitate coupling kinetic and continuum models based on continuity of half-fluxes at the boundaries [5]. The UFS uses Cartesian grid in physical space, which is generated automatically around objects embedded in computational domain. A continuum solver is running first and the computational grid in physical space is dynamically adapted to the solution. Kinetic domains are identified using continuum breakdown criteria, and the Boltzmann solver replaces the continuum solver where necessary. The Boltzmann solver uses Cartesian mesh in velocity space and employs efficient conservative methods of calculating the collision integral developed by Tcheremissine [6]. The parallel version of the UFS enforces dynamic load balance among processors. Domain decomposition among processors is performed using spacefilling curves (SFC) with different weights assigned to kinetic and continuum cells depending on CPU time required for performing computations in the cell [7].

This paper presents illustrative examples of UFS applications for supersonic and subsonic problems and outlines directions for future development of UFS methodology for simulating complex multi-scale flows.

\section{Examples of Multi-scale Simulations with UFS}

The UFS has already been demonstrated for a variety of steady-state laminar flows with different Knudsen and Mach numbers [5]. In this Section, we present some examples to illustrate important features of the UFS.

\subsection{High Speed Flows}

A comparison of UFS results with available experimental data for supersonic flows of single component atomic gases was performed for a 1D shock wave structure and 2D flow around a circular cylinder [5]. Here we show some new results for hypersonic flows and extensions for gas mixtures. Figure 1 shows an example of $2 \mathrm{D}$ solutions by

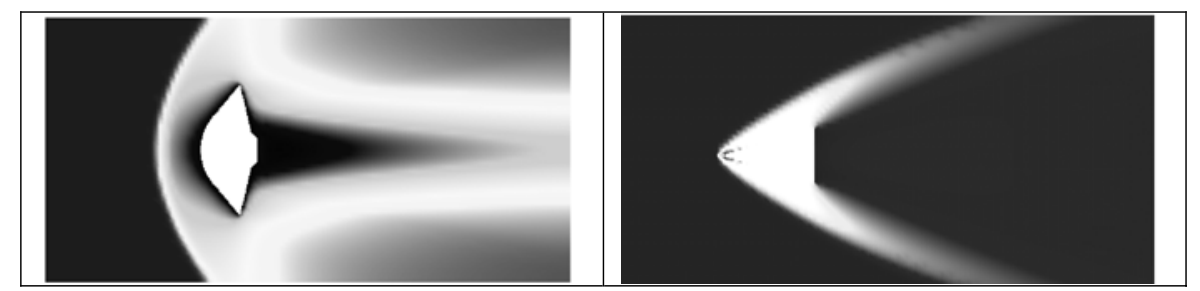

Fig. 1. Axial velocity for OREX at $\mathrm{M}=27, \mathrm{Kn}=0.1$ (on the left). Gas pressure for a prism at $\mathrm{M}=18, \mathrm{Kn}=0.25$ (on the right). 
the kinetic Navier-Stokes solver for the Orbital Reentry Experiment [9] (left) and flow around a prism (right). Kinetic scheme for the NS equation is derived from the Boltzmann equation as described in $[8,5]$

Figure 2 illustrates interaction of supersonic jets of two gases with different masses. The kinetic Euler solver for gas mixtures is based on the model of Ref. [10]. Two free jets at Mach number $M=3$ are considered with the ratio of inlet pressure to bulk pressure of 10 , and the mass ratio of 2 . The distributions of gas density and longitudinal velocity are shown in Fig. 2 for a steady state. An asymmetry with respect to $\mathrm{y}=0$ can be explained by the different masses of molecules in the top and below jets.
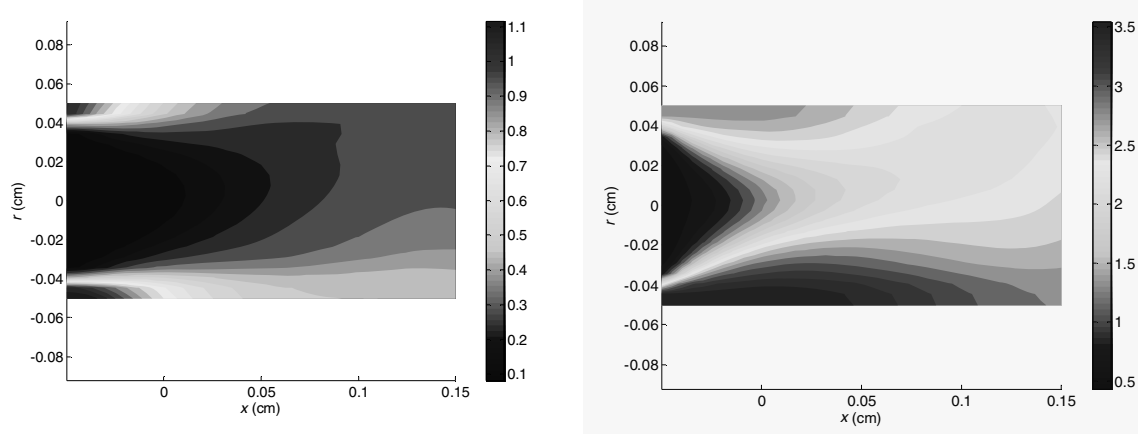

Fig. 2. Density (left) and longitudinal velocity (right) for a steady regime

The UFS is currently being enhanced with non-equilibrium chemistry (based on the model kinetic equation by Spiga \& Groppi [11]) coupled to radiation transport and plasma capabilities. This will bring the fidelity of modeling high speed flows of molecular gases to a next level and enable accurate prediction of aerothermal environment around trans-atmospheric vehicles.

\subsection{Low Speed Flows}

There is a class of low-speed continuum flows, which are not described by the traditional NS equations [12]. The Ghost and non-NS effects present themselves in well-known classical fluid problems such as Benard and Taylor-Couette problems. These effects can have important practical implications for low-pressure industrial processing reactors [13]. The statistical DSMC methods are not well suited for simulation of low speed problems, especially for studies of instabilities and transient effects due to large statistical noise.

Figure 3 illustrates a low-speed flow induced by temperature gradients between two non-uniformly heated plates. This flow is absent according to the traditional NS equations with slip boundary conditions. Both the direct Boltzmann solver and the kinetic NS solver produce correct physical picture of the flow shown in Fig. 3. The temperature $\mathrm{T}$ of surfaces goes from 1.7 to 1 (hot bottom and cold top), bottom is symmetry, top and left boundaries have $\mathrm{T}=1$. 

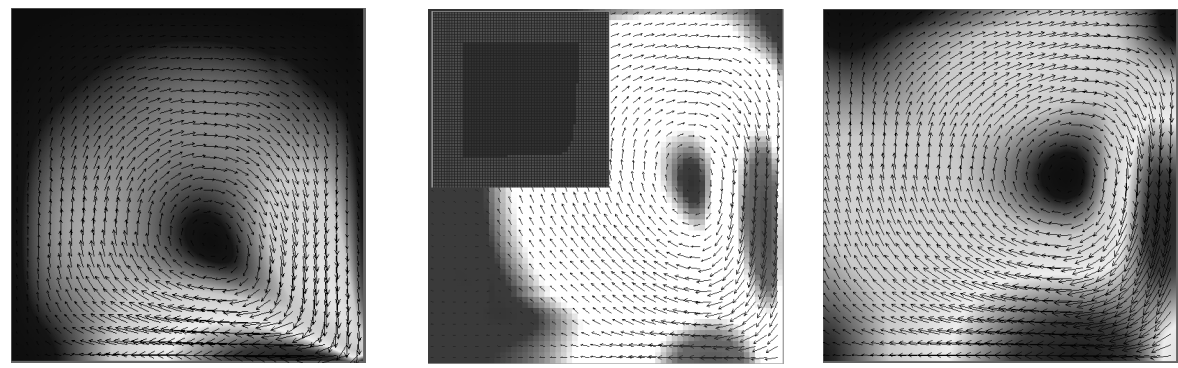

Fig. 3. Temperature driven vortex: temperature and velocity fields for three values of Knudsen numbers $(\mathrm{Kn}=0.01,0.07,0.3$ from left to right). Kinetic and continuum zones are shown in the middle Figure corner: dark - continuum, grey - kinetic zones.

The UFS has been used for simulations of gas flows in micro-channels and nozzles. We have recently confirmed the Knudsen minimum in the dependence of the mass flow rate on Knudsen number for a 2D isothermal flow in a channel using the Bolzmann solver with hard sphere molecules.

\section{Future Directions}

The Boltzmann kinetic equation provides an appropriate physical and mathematical apparatus for description of gas flows in different regimes. The UFS approach can serve the instrument for practical simulations of complex multi-scale flows including unstable and turbulent regimes. For small Kn numbers (large Re numbers) the kinetic Euler solver can be used for both laminar flows and unstable coherent large-scale structures. For smaller scales, we can select NS solver or kinetic solver according to specific criteria. In any case we assume that the kinetic solver can adequately describe micro scales for unstable and turbulent flows (but can be very expensive computationally).

From the kinetic point of view, the transition to unstable turbulent regimes can be treated as appearance of virtual structures in phase space with different scales in physical and velocity spaces. Strong deviations from equilibrium in velocity space can explain rapid growth of dissipation in turbulent flows. The applicability of NS equations in these regimes and the necessity to replace the reological linear model by more complex nonlinear models remain discussion topics [14]. The kinetic description is expected to describe correctly all flow properties at microscopic level since the smallest vortices of turbulent flows remain of the order of 100 mean free paths. The unstable flows already analyzed with the Boltzmann equation [15-17] required sufficiently fine velocity grid, but the spatial and temporal scales remained macroscopic, although smaller than for the laminar flows. For some parameters (frequencies of chaotic pulsations and the density amplitude) there was agreement with the experimental data for a free supersonic jet flows and computations from $[15-17,3]$. The capabilities of the kinetic approach have been confirmed in independent investigations of different unstable and turbulent gas flows by the DSMC schemes [18, 19] and by the BGK equation [20]. 


\subsection{Instabilities}

3D solutions of the Boltzmann equation for two Kn numbers for stable and unstable regimes are shown in Fig. 4 for a free underexpanded supersonic jet flow. The gas flows from left to right through a circular orifice, the Mach number at the orifice is $M=1$. The pressure ratio of flowing gas (to the left from the orifice) to the gas at rest (to the right from the orifice p0/p1=10. One can see in Fig. 4 that for the large Reynolds number (small Knudsen number) the gas flow is unstable. The mechanism of this instability is connected with the appearance of so-called Taylor-Gertler vortices. The Boltzmann equation has been solved by the direct conservative method with the spatial steps larger than the mean free path. The calculated system of vortices has been observed in experiments [21], and the kinetic simulations reproduced the experimentally observed macroscopic structures.
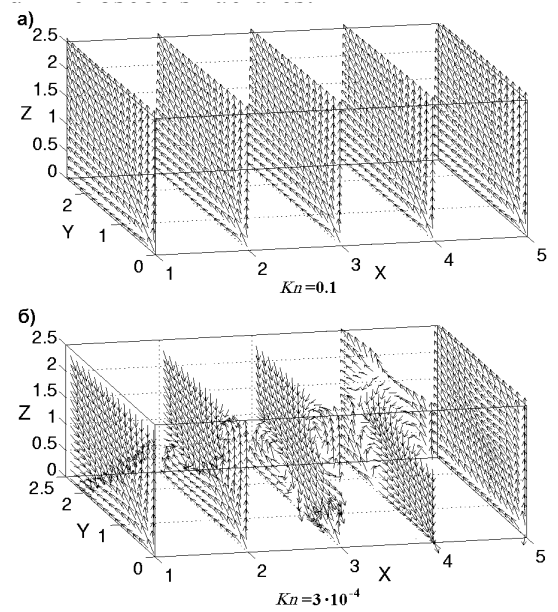

Fig. 4. Velocity fields in cross-sections for 5 values of $x$-coordinates. There is no vorticity in the plot for the larger Knudsen number, - a). Pairs of the longitudinal vortices are shown - б). A quarter for each cross section is depicted.

\subsection{Multi-scale Simulation of Turbulent Flows}

Modern approach to simulation of turbulent flows uses Euler, NS and Boltzmann models for different parts of the flows [22]. The Euler models are used for large-scale structures, whereas kinetic models are used for small-scale stochastic background. The Boltzmann equation for the velocity distribution of gas particles is used for compressive gas flows. For liquids, there is no universal kinetic equation for the probability density of instant flow velocity. Often, kinetic equation for turbulent background is used in the form resembling Boltzmann transport equation for gases.

The UFS can serve a useful tool for the first principle simulations of turbulent gas flows. The macro-scale eddies can be described by the Euler or NS equations and the Boltzmann solver used for micro-scale phenomena. For liquids, semi-empirical 
kinetic equations of the Onufriev-Lundgren type could be used at the micro-scale. Molecular dynamic simulations can help justify and improve these equations for liquids. Additional research is needed to understand how to properly expand the UFS methodology for complex turbulent flows. The macro-scale coherent structures of turbulent flows can be well described by the Euler or NS equations. Fig. 5 shows an example of 2D UFS simulations (here the kinetic NS solver is used) of unstable phenomena appeared in the wake behind a prism at $\mathrm{M}=3, \mathrm{Kn}=10^{-5}$ for the angle of attack of 3 degrees.
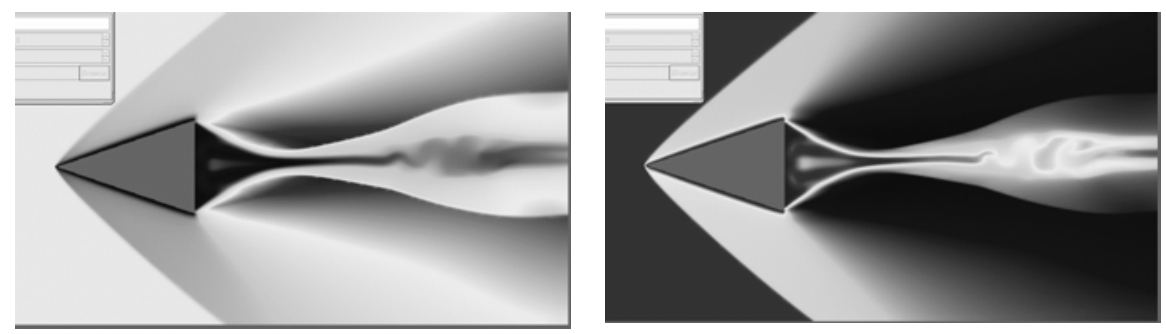

Fig. 5. Instantaneous Mach number (left) in a range $910^{-3}<\mathrm{M}<5$ and temperature (right) in a range $0.4<\mathrm{T}<4.14$ for a gas flow around a prism

\subsection{Multiscale Flow Structures in Open Systems}

The UFS can be extended for multi-scale analysis of chemical and biological structures. An important kinetic model of such problems is the nonuniform relaxation problem [23,3]. Very interesting physical effects have been observed in such systems, e.g. the heat flux has the same sign as the temperature gradient. This model could describe properties of complex natural objects [24]. Biological structures are open flux systems, which often possess two key processes: convection and chemical reactions. The scales of these processes are different: reactions are usually slower than advection. The analysis of 1D nonuniform relaxation problem for monatomic onecomponent gas has shown that nonequilibrium in velocity space is "projected" onto physical space and results in spatial structures. A generalization for mixtures showed [25] that it is possible to change the character of the spatial structures by changing the nonequilibrium distribution functions for gas components at the boundaries.

\section{Conclusion}

We have described a Unified Flow Solver for simulations of complex multi-scale gas (and possibly liquid) flows in different regimes. We considered some examples of supersonic and subsonic flows including unstable complex regimes for which the UFS and its components show reliable results. Perspectives of future development of the UFS methodology have been outlined. New approach to some important problems, such as non-uniform nonequilibrium structures for complex flows in open systems has been discussed. 
Acknowledgments. This work is partially supported by the Program N15 of the Presidium of the Russian Academy of Sciences and by the US Air Force SBIR Project F33615-03-M-3326.

\section{References}

1. Kolobov, V.I, Bayyuk, S.A., Arslanbekov, R.R., Aristov, V.V., Frolova, A.A., Zabelok, S.A.: Construction of a Unified Continuum/Kinetic Solver for Aerodynamic Problems. AIAA Journal of Spacecraft and Rockets 42, (2005) 598-605

2. Carlson, H.A., Roveda, R., Boyd, I.D., Candler, G.A.: A Hybrid CFD-DSMC Method of Modeling Continuum-Rarefied Flows. AIAA Paper 2004-1180 (2004)

3. Aristov, V.V.: Direct Methods for Solving the Boltzmann Equation and Study of NonEquilibrium Flows. Kluwer Academic Publishers, Dordrecht (2001)

4. Tcheremissine, F.G.: Direct Numerical Solution of the Boltzmann Equation In: M.Capitelli (ed.): Rarefied Gas Dynamics. AIP Conference Proceedings, Vol. 762. Melville. New York (2005) 677-685

5. Kolobov, V.I., Arslanbekov, R.R., Aristov, V.V., Frolova, A.A., Zabelok, S.A.: Unified Solver for Rarefied and Continuum Flows with Adaptive Mesh and Algorithm Refinement, J. Comput. Phys. (2006), doi:10.1016/j.jcp.2006.09.021

6. Tcheremissine, F.G.: Solution of the Boltzmann Kinetic Equation for High-Speed Flows. Comp. Math. Math. Phys. 46 (2006) 315-327

7. Zabelok, S.A. et al.: Parallel Implementation of the Unified Flow Solver, $25^{\text {th }}$ Intern Symp. on Rarefied Gas Dynamics. Book of Abstracts. St. Petersburg (2006) 62

8. Li, O., Fu, S., Xu, K.: A Compressible Navier-Stokes Flow Solver with Scalar Transport. J. Comput.Phys. 204 (2005) 692-709

9. Moss, J.N., Gupta, R.N., Price, J.M.: DSMC Simulations of OREX Entry Conditions. In: Shen, C. (ed.): Rarefied Gas Dynamics. Peking University Press, Peking (1997) 459-464

10. Andries, P., Aoki, K., Perthame, B.: J. Stat. Phys. 106 (2002) 993-1018.

11. Groppi, M., Spiga, G. A BGK Model for a Mixture of Chemically Reacting Gases. In: M.Capitelli (ed.): Rarefied Gas Dynamics. AIP Conference Proceedings, Vol. 762. Melville. New York (2005) 842-847

12. Sone, Y.: Molecular Gas Dynamics. Birkhauser, Boston (2007)

13. Arslanbekov, R.R., Kolobov, V.I.: Simulation of Low Pressure Plasma Processing Reactors: Kinetics of Electrons and Neutrals, $25^{\text {th }}$ Intern. Symp. Rarefied Gas Dynamics. Book of Abstracts. St. Petersburg, (2006) 63

14. Astarita, J., Maruchhi, L.: Basis of Hydromechanics of Non-newtonian Fluids. Mir, Moscow (1978)

15. Aristov, V.V.: Numerical Analysis of Free Jets at Small Knudsen Numbers.: In: Harvey, J., Lord, G. (eds.): Rarefied Gas Dynamics. Oxford University Press, Oxford, Vol.2. (1995) 1293-1299

16. Aristov, V.V.: Stability and Instability Analysis of Free Jets Based on the Boltzmann Equation. Fluid. Dynam. 33 (1998) 280-283

17. Aristov, V.V.: Solving the Boltzmann Equation at Small Knudsen Numbers.: Comp. Math. Math. Phys. 44 (2004) 1069-1081

18. Cercignani, C., Stefanov, S.: Vorticities and Turbulence in Hypersonic Rarefied Flows. In: Harvey, J., Lord, G. (eds.): Rarefied Gas Dynamics. Oxford University Press, Oxford, Vol.2. (1995) 1147-1153 
19. Bird, G.A. The Initiation of Centrifugal Instabilities in an Axially Symmetric Flow. In: Shen, C. (ed.): Rarefied Gas Dynamics. Peking University Press, Peking (1997) 149-154

20. Sakurai, A., Takayama, S.: Vorticity and Shock Wave in a Compressible Turbulent Flow of Complanar Kinetic Gas Model. In: Shen, C. (ed.): Rarefied Gas Dynamics. Peking University Press, Peking (1997) 291-296

21. Arnette, S.A., Samimy, M., Elliot, G.S.: On Streamwise Vortices in High Reynolds Number Supersonic Axisymmetric Jets. Phys. Fluids. A 5 (1993) 187-202

22. Belotserkovskii, O.M., Oparin, A.M., Chechetkin, V.M.: Turbulence: New Approaches. Cambridge International Science Publishing, Boston (2005)

23. Aristov, V.V.: A Steady State, Supersonic Flow Solution of the Boltzmann Equation. Phys. Letters A. 250 (1998) 354-359

24. Aristov, V.V.: Dissipative Structures Describing by the Boltzmann Equation and Relaxation Model Kinetic Equation. In: Topics in Biomathematics. World Scientific, Singapore (1993) 109-112

25. Aristov, V.V.: Nonuniform Relaxation Problem for Mixtures and Effects of Anomalous Heat Transfer. In: M.Capitelli (ed.): Rarefied Gas Dynamics. AIP Conference Proceedings, Vol. 762. Melville. New York (2005) 288-293 